\title{
Experimental study on the influence of background music on pedestrian movement in high densities
}

\author{
Guang Zeng ${ }^{1,2}$, Andreas Schadschneider ${ }^{2}$, Jun Zhang ${ }^{1}$, Weiguo Song ${ }^{1}$ \\ ${ }^{1}$ State Key Laboratory of Fire Science, University of Science and Technology of China \\ Jinzhai Road 96, Baohe district, Hefei 230027, China \\ ${ }^{2}$ Theoretical Physics Institution, University of Cologne \\ ZuelpicherStrasse 77,Cologne50937, Germany \\ gzeng@mail.ustc.edu.cn; as@thp.uni-koeln.de; \\ junz@ustc.edu.cn; wgsong@ustc.edu.cn
}

\begin{abstract}
It is interesting to investigate the effect of background music on pedestrian movement. This paper investigates the properties of crowd motion with external rhythms. With rhythm, pedestrians stop more frequently than without any rhythm. The stopping also increases with the increment of the tempo. Velocity and flow with rhythms are lower than that without any rhythm at high densities due to the more frequent stopping. Stepping behavior analysis shows that the step frequency with rhythms is smaller than that without any rhythm, especially at high densities. Dynamic coordinated behavior is weakened by music, which also affectsthe stepping behavior. Our study will be helpful for understanding the effect of background music on pedestrian movement.
\end{abstract}

Keyword:background music; stop-and-go; fundamental diagrams; stepping behavior

\section{Introduction}

Although crowding is often observed especially in large cities, it is still not clear how to control crowd motion effectively. Yanagisawa et al.[1]found that a rhythm below the normal step frequency can improve pedestrian flow if pedestrians are instructed to follow the rhythm. Therefore it is interesting to investigate the influence of rhythm on pedestrian motion in more detail, e.g. the effect of rhythm without explicit instructions or of background music.

With instruction of following the rhythm, Styns et al.[2] analyzed isolated pedestrians movement and found that the velocity will increase with the increment of rhythm tempo until 114 BPM and then keep steady. Yanagisawa et al.[1] proposed and verified that pedestrians following the rhythm slower than pedestrian normal step frequency, can improve pedestrian flow. It was explained by cognition science that the rhythm could promote cognitive processes, which results in coordinated pedestrian flow motion[3].However, there is few work about the influence of background music on pedestrian movement, especially at high densities. In a city environment, different types of music have different effects on pedestrian velocity: a pedestrian walks more quickly when listening to motivational music, while he/she walks more slowly when listening to non-motivational music[4]. Mendonca et al.[5]found that the step frequency is not influenced by music when a pedestrian walks on a treadmill. Sejdic et al.[6]also found that music does not have a natural influence on the walking behavior of pedestrians when a pedestrian walks on the ground.The effect of background music on pedestrian movement is not clear. How different tempos of rhythms influence pedestrian movement when pedestrians are not instructed to follow the rhythm, has not been investigated so far. In this study, we 
focus on the effect of differentrhythms(different tempos) on pedestrian dynamics. A series of single-file experiments, six experiments with six different rhythms and one without any rhythms, are conducted to study pedestrian movement influenced by different rhythms. These rhythms include 90BPM, 120BPM and 150BPM rhythms from a pure music of a song called "Fade" and 90BPM, 120BPM and 150BPM rhythms produced by electronic metronome.

Single-file experiments, in which pedestrians are only influenced by the purely longitudinal interactions, are commonly used to study pedestrian dynamics. Seyfried et al.[7]studied the fundamental diagram and explained the linear relation between headway and velocityin the view of stepping. Jelic et al.[8]carried out a single-file experiment in a circle corridor and found there are three different regimes in the relation between headway and velocity. Cao et al.[9] found three different regimes of the relation in mixed age group, but only two in young group by analyzing pedestrian movement.

The reminder of the paper is organized as follows: in the second part, the experiment setup will be introduced in detail. The results of the experiment and the analysis will be presented in the third part. At last, the paper will be summarized with a conclusion.

\section{Experiment setup}

To investigate the effect of background music on pedestrian dynamics, we conducted a series of single-file experiments in Hefei, China. The sketch of the scenario is shown in Fig.1. The scenario is an oval ring corridor with a central circumference of $21.93 \mathrm{~m}$ andtwo $5 \mathrm{~m}$ long straight corridors. The width of the corridor is $0.8 \mathrm{~m}$, which is wide enough for pedestrians to walk freely, but does not allow overtaking. 40 college students (18 male and 22 female) took part in the experiment. Different runs with $2,5,10,20,30$ and 40 participants were conducted to realize different global (one-dimensional) densities ranging from $0.09 / \mathrm{m}$ to $1.82 / \mathrm{m}$. In each run, pedestrians were given the same instructions to walk as normally without overtaking preceding pedestrians. They walked 5 rounds or at least $3 \mathrm{~min}$ each run to ensure that enough data for stationary movement can be extracted. Six experiments with different rhythms and an experiment under normal conditions (without any rhythms) were designed for the experiment. The former were carried out with music of 90 BPM, 120 BPM and 150 BPM and 90 BPM, 120 BPM and 150 BPM with a rhythm from an electric metronome, respectively. The music is pure music of a song called "Fade" whose original music tempo is about $108 \mathrm{BPM}$. We transformed it to three different tempos (90 BPM, 120 BPM and 150 BPM) with Adobe Audition CS6. To avoid interference of these different conditions, different runs for the same rhythm condition were conducted consecutively before the rhythm condition was changed. A total of 77 runs of the experiment were performed.For more details of the experiment we refer to[10].

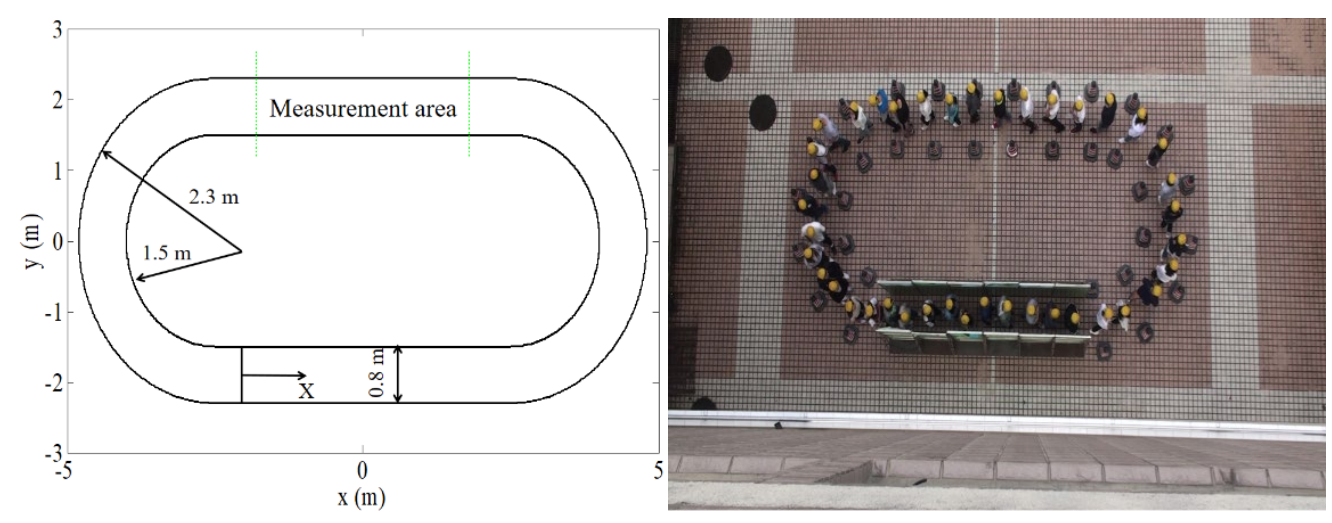

Fig.1 Schematic illustration of the scenario and a snapshot from the experiment 


\section{Results and analysis}

To analyze the data, the 2D coordinate is transformed into a $1 \mathrm{D}$ coordinate in Fig. 1 . The origin 0 of the $1 \mathrm{D}$ coordinate is at the center of the starting line of the straight corridor. The maximum length of the coordinate is $21.93 \mathrm{~m}$. The coordinate below will be described with the $1 \mathrm{D}$ coordinate, if there is no special explanation. To compare the results with different rhythms, only the data in the steady state were analyzed.

Instantaneous velocity is the average velocityin a short time interval.A Voronoi headway is defined as half of the distance between the centers of his following pedestrian and followed pedestrian. Local density is the inverse of the Voronoi headway. They can be calculated as Equation 1. Fundamental diagrams are measured in the measurement area $(11.67 \mathrm{~m}, 15.27 \mathrm{~m})$ which is shown in Fig.1. Macroscopically, Density and velocity in the measurement area are calculated as Equation 2.

$\rho(\mathrm{X}, \mathrm{t})=\frac{1}{\frac{X_{i+1}(t)-X_{i}(t)}{2}}, \mathrm{v}(\mathrm{X}, \mathrm{t})=\frac{X_{i}\left(t+\frac{\Delta t}{2}\right)-X_{i}\left(t-\frac{\Delta t}{2}\right)}{\Delta t}$

$\rho(\mathrm{t})=\frac{\int \rho(X, t) d X}{l_{m}} \quad, \quad \mathrm{v}(\mathrm{t})=\frac{\int v(X, t) d X}{l_{m}}$

Here $\rho(X, t), v(X, t), \rho(t)$ and $v(t)$ represent local density, instantaneous velocity, average density and average velocity, respectively. $\mathrm{X}_{\mathrm{i}}(\mathrm{t})$ is the $1 \mathrm{D}$ coordinate of pedestrian $\mathrm{i}$ at time $\mathrm{t} . \Delta \mathrm{t}$ is a time interval chosen here as $0.4 \mathrm{~s} . l_{m}$ represents the length of the measurement area.

\subsection{Time-space diagram}

Time-space diagram can show characteristics of pedestrian movement. To make it more clearly, we define moving and stopping here. When instantaneous velocity of a pedestrian is beyond $0.1 \mathrm{~m} / \mathrm{s}$, the pedestrian is regarded as moving, otherwise, he is stopping. Time-space diagrams at high densities $(1.37 \mathrm{ped} / \mathrm{m}$ and $1.82 \mathrm{ped} / \mathrm{m})$ are shown in Fig. 2, where the red and blue line represent the moving and stopping state, respectively. We can find that stop-and-go starts to appear apparently when the density is $1.82 \mathrm{ped} / \mathrm{m}$ (corresponding to the runs with 40 pedestrians) with and without rhythms. However, it is obvious that pedestrians stop more frequently under rhythm conditions.

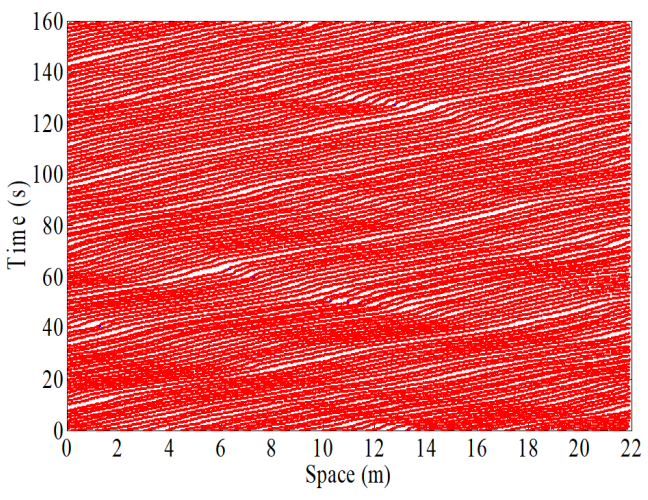

(a) Normal-30

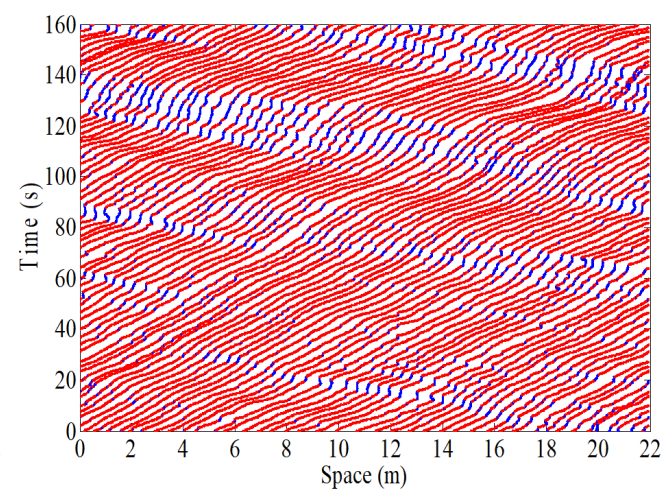

(b) Normal-40 


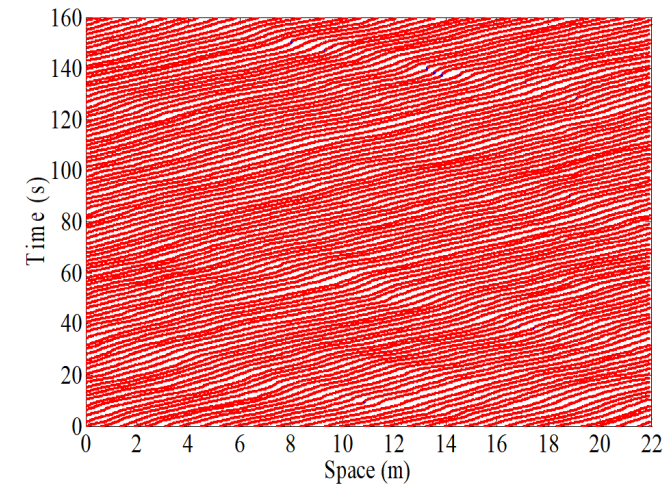

(c) Music90-30

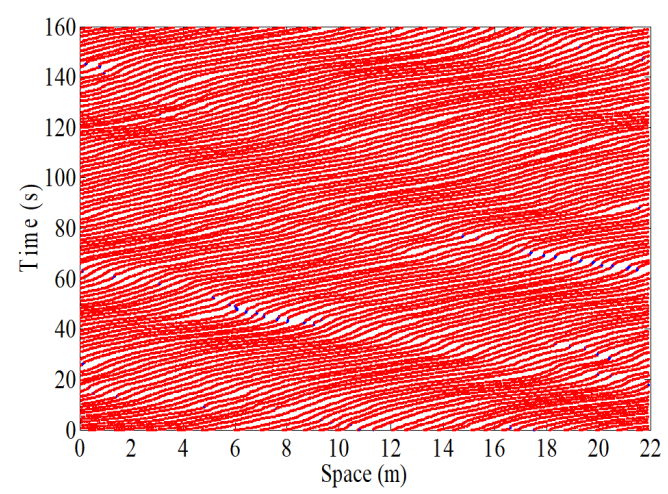

(f) Music120-30

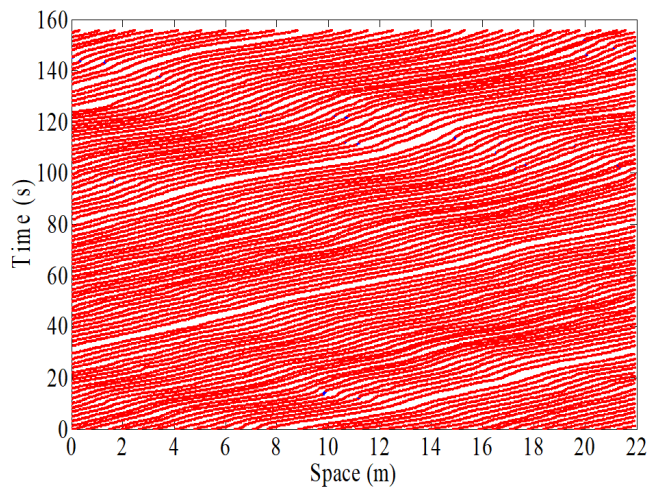

(h) Music150-30

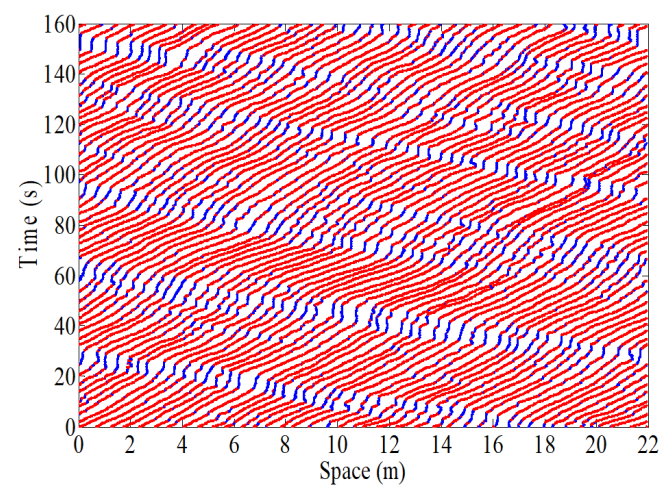

(d) Music90-40

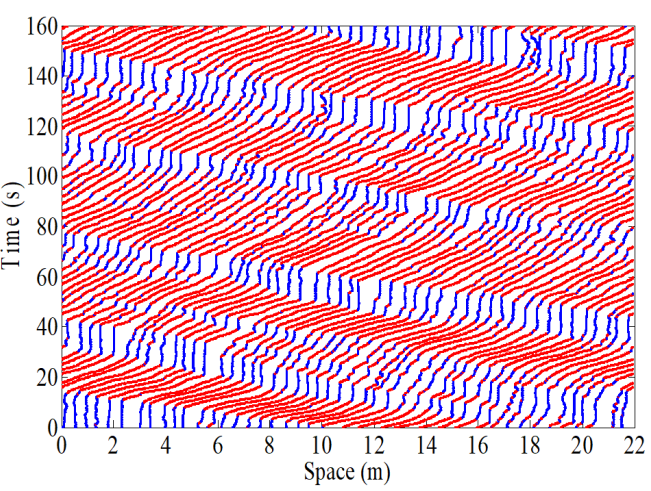

(g) Music120-40

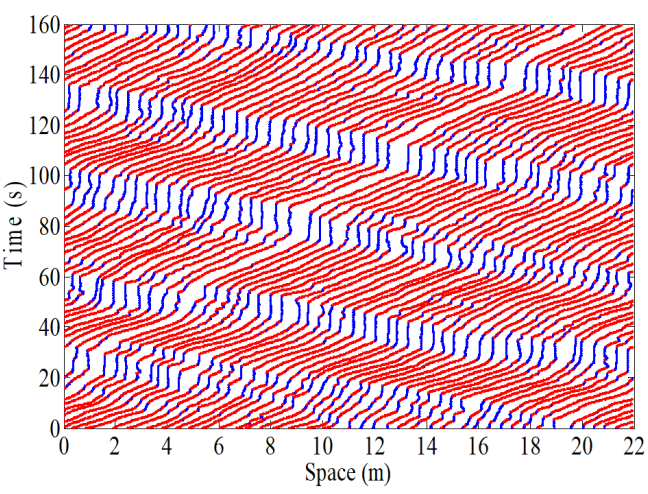

(i) Music150-40

Fig.2: Time-space diagrams for high densities. The red and blue line represent moving and stopping, respectively. Normal-30 represents the run of 30 pedestrians without any rhythm. Music90-30 means the run of 30 pedestrians with rhythm of 90BPM.

Stopping duration is defined as thelength of the time interval during which a pedestrian is not moving. It allows to compare pedestrian movement with rhythms and without any rhythm quantitatively. The distributions of stopping duration with rhythms and without any rhythm are shown in Fig.3. Considering the noise and the influence of stopping, we focus on the stopping duration longer than $0.4 \mathrm{~s}$. The ratio beyond 3.6s without any rhythm is $1.23 \%$, while those are $3.88 \%, 12.64 \%, 11.67 \%$ with 90BPM, 120BPM and 150BPM, respectively. We can find that the ratios of stopping duration beyond 3.6s with rhythms are higher than that without any rhythm. Meanwhile, that with 120BPM 
rhythm ishigher than that with 90BPM rhythm. With rhythms, pedestrians stop longer and the stopping duration increases with the tempo.

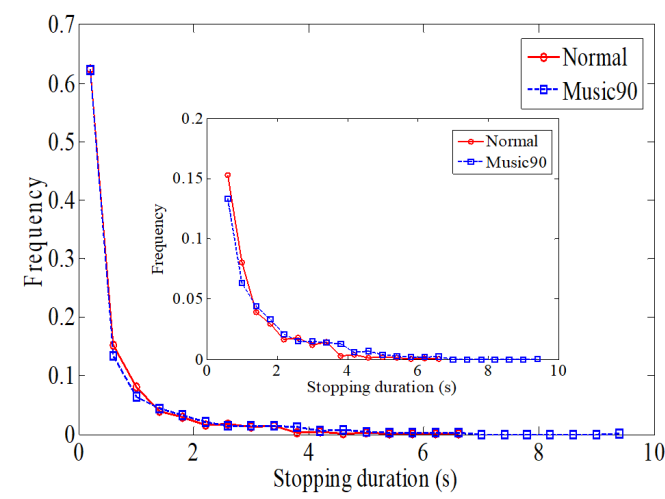

(a) Normal-Music90

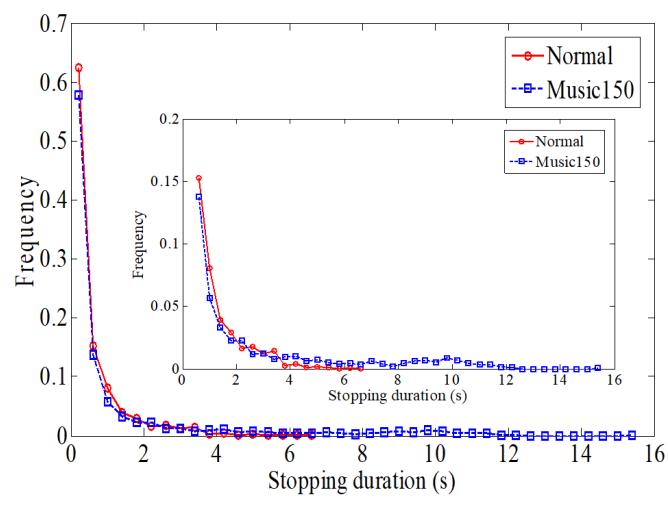

(c) Normal-Music150

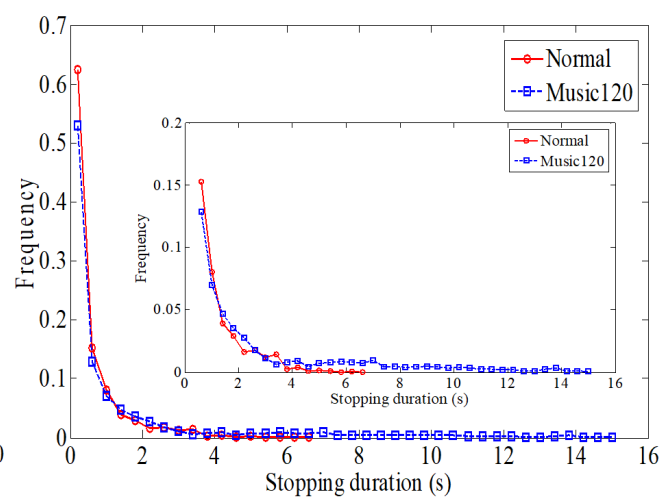

(b) Normal-Music120

Fig.3:Distribution of stopping duration with rhythms and the normal condition. The inset shows the part of distribution longer than $0.4 \mathrm{~s}$.

Stopping headway and restarting headway are defined to analyze the reason for this behavior in more detail. The headway is the distance between the centers of a pedestrian and the frontal pedestrian. Stopping headway is the headway at the frame when pedestrians start to stop. Restarting headway is the headway at the frame when pedestrians start to move again. It shows that pedestrians startto stop at a bigger headway (F-test, $\mathrm{P}<0.001$ ) with rhythms. They also start to move after stopping at a bigger headway (F-test, $\mathrm{P}<0.001$ ) in Table 1. It is the reason for pedestrians stopping more frequently with rhythms and the longer stopping duration. The restarting headway with 90BPM rhythm is bigger than that without any rhythm, but less than that with higher tempo rhythms. This may explain the stopping duration increases with the increment of the tempo.

Table 1: Stopping headway and restarting headway with rhythms and without any rhythms

\begin{tabular}{|c|c|c|}
\hline Condition & Stopping headway $(\mathrm{m})$ & Restarting headway $(\mathrm{m})$ \\
\hline Normal & $0.50 \pm 0.11$ & $0.53 \pm 0.11$ \\
\hline Music-90 & $0.51 \pm 0.10$ & $0.54 \pm 0.10$ \\
\hline Music-120 & $0.51 \pm 0.11$ & $0.55 \pm 0.11$ \\
\hline Music-150 & $0.51 \pm 0.11$ & $0.56 \pm 0.12$ \\
\hline
\end{tabular}

\subsection{Fundamental diagram}


In this section, we will focus on the fundamental diagrams. The tendency of fundamental diagrams with rhythms and without any rhythms are similar(Fig.4). However, we can also find that velocity and flow with rhythms are lower than that without any rhythm at high densities. To make it more clearly, binning procedures $[8,9]$ are made by dividing density into intervals per $0.1 / \mathrm{m}$ from $0 / \mathrm{m}$ in Fig.6. Mean value and standard deviation in every interval are calculated. It shows that velocity and flow with rhythms are smaller than that without any rhythmat high densities. With rhythms, velocity and flow with 120BPM rhythm are less than that with other rhythms in high densities. Compared to mean value of velocity(per $0.1 / \mathrm{m}$ ) at high densities(from $1.5 / \mathrm{m}$ to $2.1 / \mathrm{m}$ ), it decreases $15 \%, 42 \%$ and $21 \%$ on average with 90BPM, 120BPM and 150BPM, respectively. The increase of stopping results in the decrease of velocity and flow at high densities.

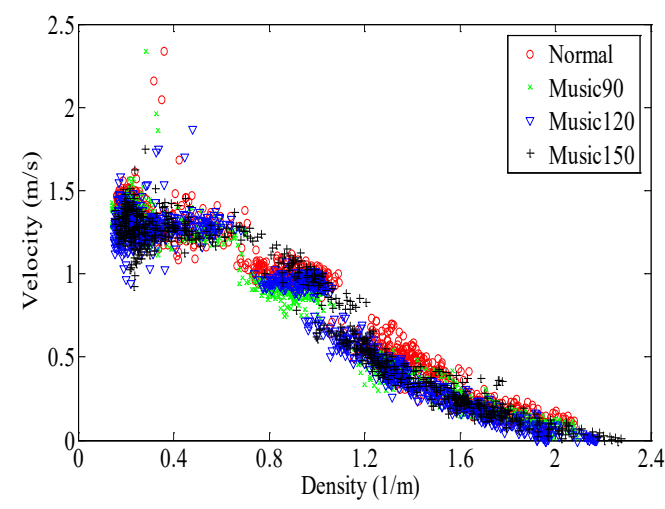

(a) Normal-Music

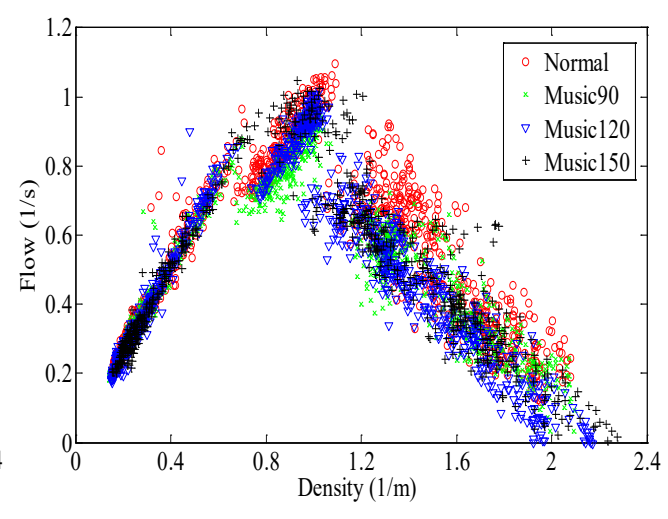

(b) Normal-Music

Fig.4: Fundamental diagrams with rhythms and without any rhythms

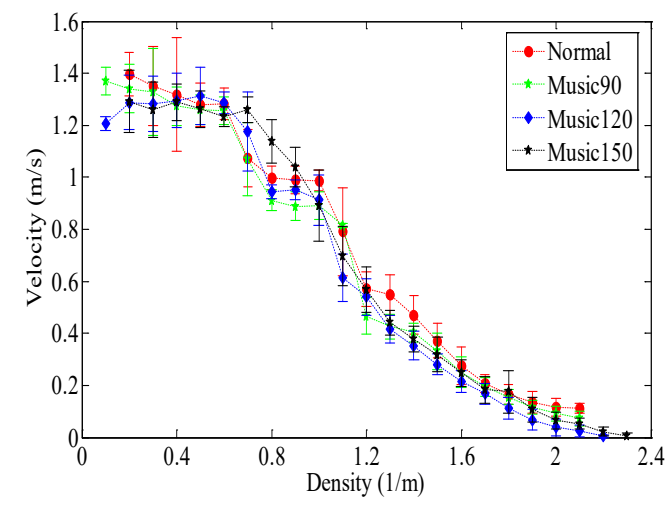

(a) Normal-Music90

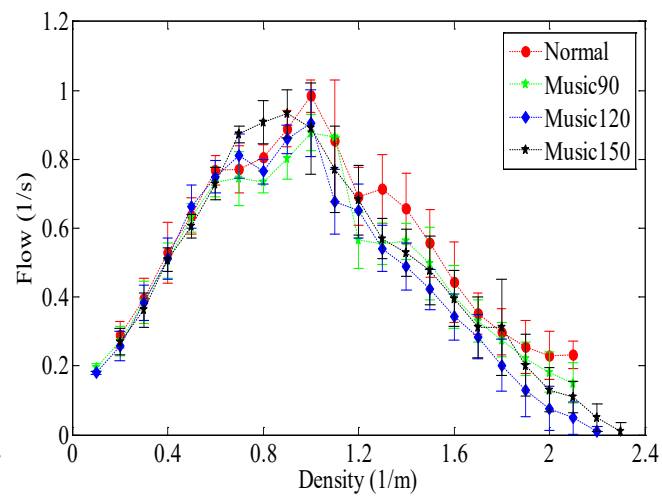

(b) Normal-Music90

Fig.5: Binning procedures fundamental diagrams with rhythms and without any rhythm.

\subsection{Stepping behavior}

Stepping is the fundamental property of pedestrians. It can be used to explain the macroscopic properties, such as the fundamental diagram. Eight or ten pedestrians were selected to count the number of steps ofeach round for runs with 2, 10, 20 and 30 pedestrians with the videos of experiments.The step is roughly defined as the process of one foot touching the ground to the other touching the ground. Step length $(s l)$ and step frequency $(s f)$ are calculated as Equation 3.

$s l=\frac{\mathrm{L}}{S N} \quad, \quad s f=\frac{T}{S N}$

Here $\mathrm{L}$ means the length of the corridor, which is $21.93 \mathrm{~m} . S N$ and $T$ represent step number and 
the time for these steps.

The results for step length and step frequency with rhythms and without any rhythms are shown in Table 2. It indicates that the step frequency with rhythms is slightlyless than that without any rhythms except for that of the run with 20 pedestrians with 150BPM rhythm at low and medium densities. At high densities, the step frequencywith rhythms decreases a lot compared to that without any rhythms. Lang et al.[11] found that music will weaken dynamic coordinated behavior, which results in finishing successive behavior with more time. Therefore, it can be explained that pedestrians need more time to step in the presence of music, especially at high densities.

Table 2: Step length and step frequency with rhythms and without any rhythms

\begin{tabular}{|c|c|c|}
\hline Condition & Step length $(\mathrm{m})$ & Step frequency(Hz) \\
\hline Normal-2 & $0.74 \pm 0.05$ & $1.97 \pm 0.10$ \\
\hline Music90-2 & $0.73 \pm 0.05$ & $1.93 \pm 0.08$ \\
\hline Music120-2 & $0.73 \pm 0.06$ & $1.94 \pm 0.09$ \\
\hline Music150-2 & $0.76 \pm 0.07$ & $1.97 \pm 0.09$ \\
\hline Normal-10 & $0.68 \pm 0.03$ & $1.92 \pm 0.08$ \\
\hline Music90-10 & $0.66 \pm 0.03$ & $1.89 \pm 0.07$ \\
\hline Music120-10 & $0.68 \pm 0.03$ & $1.91 \pm 0.08$ \\
\hline Music150-10 & $0.67 \pm 0.03$ & $1.89 \pm 0.07$ \\
\hline Normal-20 & $0.58 \pm 0.04$ & $1.63 \pm 0.10$ \\
\hline Music90-20 & $0.56 \pm 0.03$ & $1.56 \pm 0.07$ \\
\hline Music120-20 & $0.60 \pm 0.03$ & $1.62 \pm 0.09$ \\
\hline Music150-20 & $0.60 \pm 0.03$ & $1.66 \pm 0.09$ \\
\hline Normal-30 & $0.38 \pm 0.04$ & $1.38 \pm 0.15$ \\
\hline Music90-30 & $0.36 \pm 0.03$ & $1.11 \pm 0.11$ \\
\hline Music120-30 & $0.39 \pm 0.03$ & $1.10 \pm 0.11$ \\
\hline Music150-30 & $0.38 \pm 0.02$ & $1.11 \pm 0.08$ \\
\hline
\end{tabular}

\section{Conclusion}

To study the effect of background music on pedestrian movement, we conducted a series of single-file experiments. The trajectories were extracted by PeTrack[12]. With the trajectories, time-space diagrams and fundamental diagrams are analyzed.

Stop-and-go behavior appears apparently at global density 1.82/mwith rhythms and without any rhythm. However, compared with pedestrian movement without any rhythm pedestrians stop more frequently with rhythms.Stopping durationwill also be longer with rhythms andincreases with the increment of the tempo.

With rhythms and without any rhythm, there is a similar tendency in fundamental diagrams. However, velocity and flow with rhythms are lower than that without any rhythms. The mean value of velocity(per $0.1 / \mathrm{m}$ ) from $1.5 / \mathrm{m}$ to $2.1 / \mathrm{m}$ decrease $15 \%, 42 \%$, and $21 \%$ on averagefor 90BPM, 120BPM and 150BPM rhythm, respectively. Stopping contributes to the decreasing of velocity and flow.

With rhythms, dynamic coordinated behavior of pedestrians will be weakened, which will increase pedestrian stepping time. Due to it, the velocity at low density is lower than that without any rhythms. With the increasing of density, pedestrians will also be influenced by the interaction with the 
frontal pedestrians. Coupled with them, step frequency will decrease more quickly, which contributes to the sharp decrease ofvelocity and flow at high densities. When the step frequency cannot decrease any more, pedestrians will stop. This may explain that the stopping headway with rhythms is higher than that without any rhythms. The effect of rhythms will increase with the increment of tempo.

In the future, the stepping behavior needs to be investigated, which will be helpful for understanding the effect of background music more clearly.

\section{Acknowledgement}

The authors acknowledge the foundation support from Key Research and Development Program(2016YFC0802500), German Science Foundation (grant SCHA 636/9-1), the National Natural Science Foundation of China (Grant No. 71704168), the Anhui Provincial Natural Science Foundation (Grant No. 1808085MG217) and the Fundamental Research Funds for the Central Universities (Grant No. WK2320000040)and the China Scholarship Council(CSC).

\section{Reference}

[1] D. Yanagisawa, A. Tomoeda, and K. Nishinari, "Improvement of pedestrian flow by slow rhythm," Phys Rev E Stat Nonlin Soft Matter Phys, vol. 85, p. 016111, 2012.

[2] F. Styns, L. van Noorden, D. Moelants, and M. Leman, "Walking on music," Hum Mov Sci, vol. 26, pp. 769-85, 2007.

[3] S. Ikeda, T. Nozawa, R. Yokoyama, A. Miyazaki, Y. Sasaki, K. Sakaki, et al., "Steady Beat Sound Facilitates both Coordinated Group Walking and Inter-Subject Neural Synchrony," Front Hum Neurosci, vol. 11, p. 147, 2017.

[4] M. Franek, L. van Noorden, and L. Rezny, "Tempo and walking speed with music in the urban context," Front Psychol, vol. 5, p. 1361, 2014.

[5] C. Mendonca, M. Oliveira, L. Fontes, and J. Santos, "The effect of instruction to synchronize over step frequency while walking with auditory cues on a treadmill," Hum Mov Sci, vol. 33, pp. 33-42, 2014.

[6] E. Sejdic, B. Findlay, C. Merey, and T. Chau, "The effects of listening to music or viewing television on human gait," Comput Biol Med, vol. 43, pp. 1497-501, 2013.

[7] A. Seyfried, B. Steffen, W. Klingsch, and M. Boltes, "The fundamental diagram of pedestrian movement revisited," Journal of Statistical Mechanics: Theory and Experiment, vol. 2005, pp. P10002-P10002, 2005.

[8] A. Jelic, C. Appert-Rolland, S. Lemercier, and J. Pettre, "Properties of pedestrians walking in line: fundamental diagrams," Phys Rev E Stat Nonlin Soft Matter Phys, vol. 85, p. 036111, 2012.

[9] S. Cao, J. Zhang, D. Salden, J. Ma, C. Shi, and R. Zhang, "Pedestrian dynamics in single-file movement of crowd with different age compositions," Phys Rev E, vol. 94, p. 012312, 2016.

[10] G. Zeng, A. Schadschneider, J. Zhang, S. Wei, W. Song, and R. Ba,"Experimental study on the effect of background music on pedestrian movement at high density," Physics Letters A.

[11] M. Lang, D. J. Shaw, P. Reddish, S. Wallot, P. Mitkidis, and D. Xygalatas, "Lost in the Rhythm: Effects of Rhythm on Subsequent Interpersonal Coordination," Cogn Sci, vol. 40, pp. 1797-1815, 2016.

[12] M. Boltes and A. Seyfried, "Collecting pedestrian trajectories," Neurocomputing, vol. 100, pp. 127-133, 2013. 